\title{
Des écrivains et leur rapport à la Première Guerre mondiale : traces littéraires de la Grande Guerre dans la littérature produite en Alsace (allemand, parler dialectal alsacien) entre 1914 et 1939
}

\section{Dominique Huck}

Université de Strasbourg

\section{Abstract}

\begin{abstract}
Das Ende des Krieges bedeutet für das Elsass nicht nur endlich Waffenruhe, sondern auch einen politischen Wechsel, da das Reichsland Elsass-Lothringen wieder Frankreich angehört. Dieser Umschwung schlägt sich im Leben aller Menschen nieder, beruflich, sprachlich, kulturell und sozial. Die Frage stellt sich nun, ob er auch im literarischen Wirken ein Echo findet, ganz besonders als schriftstellerischer Stoff. Um der Frage nachzugehen, fokussiert sich das Interesse auf Autoren, deren Ruf nicht viel weiter als die eigene Region geht und/oder die eher in der Mundart schreiben, da das Werk berühmter deutschschreibender Schriftsteller bereits schon diesbezüglich untersucht wurde. Der Krieg wurde eigentlich literarisch wenig thematisiert, so wie die soziopolitischen Umwälzungen, die danach stattgefunden haben. Die Schreibenden haben sich eher in ein emotionsgeladenes Elsass geflüchtet, das als Matrix ihrer Identität fungiert.
\end{abstract}

En Alsace, la fin de la guerre a amené un soulagement général de la population, comme partout ailleurs, mais a aussi entraîné un changement d'appartenance étatique dans la mesure où la « Terre d'Empire » est (re)devenue française. Ces événements touchent chaque individu dans sa vie et ses habitudes, professionnelles, linguistiques, culturelles, sociales, ... La guerre et les grands bouleversements qui en sont issus se manifestent-ils aussi dans la production littéraire, notamment sur un plan thématique ? On ne retiendra ici ni les écrivains les plus célèbres, ni ceux qui ont quitté l'Alsace après 1918, mais ceux dont la renommée reste plutôt régionale ou locale, notamment ceux qui utilisent un parler dialectal comme moyen d'expression. De fait, peu d'auteurs ont thématisé la guerre et, encore moins, les bouleversements sociopolitiques qui ont suivi. La « trace » est bien plus émotionnelle, sous la forme d'une affirmation identitaire et d'un recours à une Alsace matricielle.

In Alsace, the end of the First World War brought a great sense of relief to the population, as it did everywhere else; yet, it also brought about a major political change to the former 'Empire land' (Reichsland) which became part of France (again). This affected the daily lives of each individual, in their professional, linguistic, cultural or social dimensions. Did the war and its aftermaths manifest themselves in literature, especially at a thematic level? To answer this question, we shall focus neither on the most famous writers, nor on those who left Alsace after 1918 , since their work has already been examined under that angle, but on writers whose reputation remained regional or local, in particular on those writers who turned to the Alsatian dialect as means of expression. Indeed, in this literature, the war was rarely addressed and its socio-political upheavals even more rarely so. The 'trace' left by that conflict is of an emotional nature; it takes the form of an identity statement. Such writers found refuge in an emotionally charged Alsace, which functions as a matrix of their identity.

Keywords

Première Guerre mondiale $•$ Alsace $•$ littérature régionale $\bullet$ identité

Erster Weltkrieg • Elsass • elsässische Literatur • Identität

First World War • Alsace • Alsatian literature • identity

Quelle que soit la thématique abordée pour l'Alsace de l'entredeux-guerres, il n'est guère possible de faire abstraction du contexte sociopolitique particulier. Par « contexte », il faut comprendre la complexité et l'hétérogénéité des situations, accepter qu'il est, en soi, dynamique et que ses nombreuses composantes sont non seulement inséparables les unes des autres mais qu'elles interagissent entre elles, créant encore un autre type de dynamique.

Partant, une question - centrale ? - dans la création littéraire porte sur les traces que laisseront ou non les événements qui travaillent le contexte, et tout particulièrement la Grande Guerre et les vingt années porteuses de conflits 
touchant à l'« identité » (revendiquée du moins par une majorité d'habitants) qui la suivent, dans les productions (en allemand ou en en dialecte) des auteurs vivant en Alsace, entre 1914 et 1939.

\section{PROLOGUE : LE CONTEXTE ALSACIEN ENTRE 1871 ET 1918}

Après la guerre franco-prussienne de 1870 et la défaite de la France, l'Alsace et une partie de la Lorraine deviennent, au sein du tout nouvel empire allemand, un "Reichsland», une "Terre d'Empire » appartenant à tous les États de l'Empire placée, pour son administration politique, sous la tutelle directe de Berlin. D'un point de vue linguistique au sens étroit, ce changement d'appartenance politique ne pose pas de problème insurmontable dans la partie où la langue des habitants, dans leur quotidien, est un parler dialectal alémanique ou francique, et où l'allemand standard reste la langue de la religion et de la majeure partie de l'écrit lu par la population. Le français est connu des lettrés et des couches économiquement et socialement supérieures de la population, et a été enseigné aux enfants qui sont allés à l'école à partir de la décennie 1850-1860, même s'il a souvent été « oublié » par manque d'usage. Cela ne signifie pas pour autant que les sentiments patriotiques auraient penché du côté de l'Allemagne : le fait de ne pas ou de peu savoir le français n'empêche pas un sentiment d'appartenance politique à la France. Avec les générations nées après 1870, sous l'effet de la disparition naturelle de la génération qui a entretenu le " souvenir de la France " et celui des dynamiques sociopolitiques du temps, les revendications ne portent plus sur un retour à la France, mais essentiellement sur le fait de devenir un État à part entière au sein de l'Empire, c'est-àdire d'avoir la même autonomie politique que les vingt-cinq autres États. Or, comme l'Empire ne semble pas disposé à l'accorder, les élites, notamment culturelles, vont pousser les feux pour créer l'idée d'une différence identitaire, d'une sorte d'identité propre à l'Alsace, à la fois en s'appuyant sur les us et coutumes "traditionnels " (ou catégorisés comme tels) et en donnant aux parlers dialectaux une valeur propre. Tout cela va être catégorisé comme " alsacien » / " elsassisch », catégorie qui fonctionne comme hyperonyme pour bien se démarquer de ce qui serait génériquement " allemand " / « ditsch ». Sur le plan littéraire, un mouvement important va reprendre et amplifier la création en dialecte, en donnant, par exemple, une impulsion décisive au «théâtre alsacien ". Les discussions sur l'opportunité d'employer un parler dialectal au lieu du standard pour faire littérature seront passionnées et diviseront les écrivains, mais la frontière restera très poreuse et parfois évolutive dans les faits entre les tenants des deux camps. Cependant, une partie des courants francophiles encouragera la création en dialecte en l'interprétant, assez largement à tort, comme un signal du refus d'écrire en allemand standard. L'obtention d'une Constitution propre en 1911, encore bien restrictive par rapport à celles des autres États, ne satisfera pas les partisans de l'autonomie politique. Néanmoins, à la veille du Premier conflit mondial, l'opinion publique, avec la diversité des histoires de vie de chacun, les micro-appartenances (religieuses, culturelles, professionnelles, sociales, ...) semble, dans sa majorité, ne plus se poser la question du retour à la France, mais bien vivre, sans grand état d'âme, dans le cadre impérial allemand (Wahl 2019 : 101-102). C'est à partir de la proclamation du Kriegsgefahrzustand du 31 juillet 1914 que les choses changent : ce sont les militaires qui prennent les commandes dans le Reichsland (Grandhomme 2013 : 249-326) et cherchent à " mieux » faire que l'administration civile, par des mesures tatillonnes, rigides, sans égard pour la population, en pratiquant une germanisation à outrance. Cette façon d'opérer, combinée à toutes les privations et à tous les malheurs liés à la guerre, a sans doute contribué à modifier la position de l'opinion publique à l'égard du cadre politique allemand. Néanmoins, la liesse qui s'empare des villes où entrent les soldats français en 1918 n'est pas vraiment un indicateur politique qui signifierait une volonté des habitants de (re)devenir français. C'est bien plus « une décompression, un défoulement après la longue tension qui a précédé ", " la paix libératrice, la fin de la pénurie alimentaire » ainsi que « la fin de l'oppression par les militaires » (Wahl 2019 : 103).

\section{LA SPÉCIFICITÉ DU VÉCU DE LA GUERRE ET DE SES CONSÉQUENCES DANS LA SOCIÉTÉ ALSACIENNE : " LES " ALSACIENS ET LEURS LOYAUTÉS}

Si la population semble exprimer un immense soulagement à la fin de la guerre, comme partout ailleurs dans les pays belligérants, le retour à la vie d'« avant » semble bien complexe. Dans l'immédiat, pour ceux qui avaient été soldats (380 000 Alsaciens et Lorrains ${ }^{1}$ ), « leur témoignage n'est tout simplement pas recevable dans une société alsacienne et lorraine mosellane désormais officiellement déliée de son passé germanique » (Grandhomme 2013 : 439) et ils sont réduits au silence, en dehors de leur cercle privé.

Globalement, la majorité des Alsaciens n'a probablement pas envisagé qu'elle allait (re)devenir française, c'est-à-dire changer de cadre politique, culturel, idéologique et structurel de référence (cf. les témoignages chez Grandhomme 2013 : 156-157). La France va, de fait, choisir la " patrie » des habitants de l'Alsace, en leur lieu et place, en les classant selon des critères généalogiques, en gardant les " vrais " Alsaciens et en expulsant les autres, par le biais de commissions de triage (ibid. : 411-419). Ces anciens Allemands (1870-1918) deviendront donc des Français, du moins ceux qui ont le « bon » profil. Puis, la francisation de l'ensemble de la vie publique, le déclassement social et professionnel de ceux qui ne savent pas ou mal le français, la méconnaissance des réalités du fonctionnement de la société de

50000 ne reviendront pas de la guerre et 150000 sont blessés (Grandhomme $2013: 81$ ). 
la part de la France, les profondes divergences sur la question de la séparation de l'Église et de l'État, etc. amèneront une " une crise politique » majeure (Baas 1972 : 9) entre la France et une majorité d'Alsaciens : le " malaise alsacien » (1918-1924).

Dans ces conditions, la guerre et ses suites immédiates ont un impact bien différent encore qu'ailleurs dans la vie même des habitants : d'une certaine manière, elle rebat les cartes du destin de chaque habitant, qu'il s'en réjouisse ou qu'il le déplore, dans ses convictions, dans sa façon de vivre, dans ses langues, ... Le rôle de la guerre est ainsi multiple, mais essentiel pour la vie de ceux qui habitent cet espace.

\section{LA CRÉATION LITTÉRAIRE DANS CE CONTEXTE}

\subsection{Les « grands » auteurs}

Dans cette logique, la question du choix même des écrivains à retenir se pose notamment en ce qui concerne les auteurs qui ne continueront pas à vivre en Alsace après 1918 - volontairement ou non - et/ou qui ne revendiquent pas d'attache particulière avec l'Alsace, d'une part, et, d'autre part, les écrivains dont l'œuvre a largement dépassé les frontières de l'Alsace et que l'histoire littéraire retient dans les littératures nationales, comme René Schickele, Yvan et Claire Goll, Otto Flake, Ernst Stadler, Hans Arp ou, dans un autre registre, Oskar Wöhrle, pour n'en nommer que quelquesuns. Leur œuvre a été nettement plus travaillée que celle d'écrivains dont l'aura a été considérée, à tort ou à raison, comme plus modeste ou d'écrivains qui s'adressent consciemment à un public régional ou local et qui, dans leur majorité, restent dans une forme de production littéraire traditionnelle (une sorte de " Heimatliteratur »), sans lien avec les grands mouvements de leur temps (cf. Fichter 2010 : passim). Ce sont les raisons pour lesquelles les auteurs suprarégionaux ou ayant quitté l'Alsace n'ont pas été retenus, même s'ils figurent dans les anthologies citées (cf. infra).

\subsection{Les auteurs « régionaux » ou « locaux »}

C'est l'œuvre des écrivains "régionaux » ou " locaux », qui publient d'ailleurs souvent abondamment, qui sera donc examinée principalement. Ceux qui choisissent d'écrire essentiellement ou exclusivement en alsacien, en privilégiant, selon les auteurs, le théâtre ou le lyrisme, la prose étant essentiellement produite en allemand, plus rarement en alsacien, écrivent sans doute, pour la plupart, pour un public qui leur ressemble, ce qui ne signifie pas pour autant que, qualitativement, leurs productions seraient majoritairement moyennes ou médiocres, même si l'intérêt littéraire est très inégal et très fréquemment convenu, très proche d'une Heimatdichtung ou de Gelegenheitsgedichte ${ }^{2}$, avec de notables exceptions comme Nathan Katz.

C'est l'un des reproches essentiels qu'adresse (déjà !) le très nationaliste Götze dès 1917 à la production alsacienne (en langue allemande) durant la guerre pour conclure que, décidèment, « Krieg und Kunst haben unmittelbar wenig miteinander zu tun » (Götze 1917 : 29).
C'est l'importance de la quantité de textes produits et publiés qui pourrait laisser penser que, durant l'entre-deux-guerres, le conflit auquel toutes les familles ont été confrontées serait sans doute assez fréquemment évoqué ou pourrait servir de cadre pour une forme littéraire. D'autant que, dans la vie politique régionale, les suites du « grand tournant » de 1918 sont d'une actualité brûlante au quotidien et concernent toute la population au moins d'un point de vue linguistique, religieux et culturel, mais aussi d'un point de vue social, économique, et bien d'autres champs de la vie. C'est tout particulièrement vrai durant la période du « malaise alsacien ».

\section{LA PRODUCTION LITTÉRAIRE RÉGIONALE}

Abondante, la production littéraire nécessite des choix et des arbitrages. Nous avons pris le parti - sans doute discutable de retenir les quatre anthologies publiées entre 1919 et 1939 , chaque éditeur, faisant lui-même des choix esthétiques, idéologiques, quantitatifs, etc. qui lui étaient propres. C'est en confrontant les choix que se dégagent à la fois des noms et des thématiques, et plus précisément celle de la guerre et de ses suites (ou non), qu'a été établi le corpus de travail. II s'agit là d'un premier indicateur, à la fois des options thématiques et des orientations plus idéologiques des anthologies. Une dernière anthologie, " La littérature dialectale alsacienne ", en cinq volumes, parue à la fin du $X X^{e}$ siècle, retient pour les deux périodes de 1870-1918 et 1918-1945, un certain nombre d'écrivains qui ont, en quelque sorte, traversé le temps et été jugés dignes d'y figurer. Au total, il s'agit ainsi de plus d'une centaine d'écrivains dont les productions littéraires sont évoquées.

Cependant, relativement peu d'écrivains ont évoqué la guerre et ses horreurs, la vie des soldats et des civils, ses conséquences politiques, sociales, culturelles, ..., du moins, dans les textes retenus.

En revanche, si les questions d'appartenance nationale et/ ou de loyauté sont abordées de manière biaisée en 1919 ou de manière personnelle très tranchée pour la France, de nombreux auteurs prennent une position explicite et implicite autocentrée : leur appartenance première, dans la logique même des années 1900, c'est l'Alsace, c'est le fait de se sentir profondément alsacien, sans que l'on sache nécessairement ce que les uns et les autres entendent par là ${ }^{3}$.

La première anthologie qui parait dès 1919, réunie par Louis Edouard Schaeffer (1902-1988) est assez singulière : tout en indiquant qu'il l'avait pensée comme complément ou supplément à la grande anthologie de Désiré Müntzer Neues Elsässer Schatzkästlein [1913], le contenu annoncé est clairement une "poésie dialectale de nature patriotique » ( "Dialektdichtungen patriotischer Art») (p. X) et son titre en

\footnotetext{
3 Nicolas Stoskopf (2019) souligne, d'une part, que ce sentiment semble déjà ancien et que, d'autre part, il ne s'agit pas d'une invention de l'intelligentsia du tournant du siècle, en citant la correspondance que la mère de Gustave Stoskopf, adressait à son fils : " Du moment qu'ils [les Français] donnent l'hospitalité aux Anglais, Américains, etc., pourquoi faire une exception pour les Alsaciens et pourquoi leur demander le plus grand sacrifice qu'on puisse faire, celui de renoncer à jamais à leur patrie et leur fortune, car on est pourtant alsacien avant d'être français ou allemand » (lettre du 7 mars 1888, Stoskopf G. 2019 : 111).
} 
est le programme : «Blöj-wiss-rot ». Mais le jeune Schaeffer ne trouvera pas suffisamment de textes et devra partiellement se rabattre sur des productions qui ne dépareilleraient pas l'ensemble (p. X-XI). II regrette que deux noms incontournables de la littérature en dialecte n'aient pas accepté de figurer dans l'anthologie : Gustave Stoskopf (1869-1944) et les frères Albert (1874-1930) et Adolphe (1874-1944) Matthis. De fait, Gustave Stoskopf, peintre et dramaturge, l'un des cofondateurs et directeur du Théâtre Alsacien de Strasbourg, occupera de très nombreuses fonctions, notamment dans le milieu artistique, mais ne publiera presque plus de pièce de théâtre après 1918. Son refus pourrait être lié au peu d'estime que lui inspire Schaeffer ${ }^{4}$. II est plus étrange que les frères Matthis aient refusé la proposition : francophiles déclarés depuis toujours, ils avaient signé ensemble - fait rare - un texte enthousiaste Mer sin franzesch ${ }^{5}$, et ils continueront à publier régulièrement durant l'entre-deux-guerres, même après le décès d'Albert en 1930. Albert avait été soldat sur le front russe, mais aucun vers ne rappelle cet épisode. Adolphe avait été placé en résidence forcée à Altena dont il rapportera un long poème narratif Sengessle üs'm Exil (Matthis 1931 : 22-32). Mais ni l'un ni l'autre n'évoqueront la Grande Guerre en soi, ni les suites immédiates du changement politique. Dans le choix que fait Schaeffer, aucun texte n'évoque directement la guerre, mais pour l'un ou l'autre des poètes, il a néanmoins retenu un texte qui n'est pas simplement une sorte d'hymne au retour à la France, mais une évocation des malheurs que tous ont subis, sans qu'importe réellement de savoir qui est le vainqueur, comme celui de Henri Müller (1885-?) Wiehnachte 1918 :

Verschwunde wie ne Sturm, e schlimmer,

die schrecklige, die schware Zit,

Vergrawe unter Schutt un Trimmer,

verflüecht verdammt vo alle Lit. [...]

's hat sich erfillt, das Wort das scheene,

Wu 's Menschegschlàcht züem Friede mahnt,

Züem Friede, wu de freie Velker

D'r Wag züem Glick, züem Wohlstand bahnt.

Zittelboscht (p. 86-87)

Globalement, si l'on excepte cette première anthologie Blöjwiss-rot, à vocation explicitement patriotique, les trois autres anthologies publiées successivement en 1924, 1929 et 1933 n'abordent pas du tout ou, de manière très ponctuelle, la guerre en elle-même. Si Nathan Katz (1892-1981) est l'un des rares à figurer dans les trois anthologies (avec Raymond Buchert [1893-1968], Frank Oom ${ }^{6}$, Jean Sebas [1880-1971] et Henri Solveen [1891-1956]), ce ne sont pas ses textes sur la guerre ou qu'il a écrits durant la guerre qui sont retenus, en dehors de l'incontournable D'Kamerade, wu im Chrieg

$4 \quad$ Lorsque Schaeffer évoquait ses souvenirs, il rappelait que Stoskopf ne le prenait pas au sérieux et l'appelait « d'r Schaeffer in de kurze Hosse » (archives privées). Il est vrai qu'en 1919 , il n'avait que 17 ans tandis que Stoskopf, à 50 ans, était une figure établie et reconnue.

5 Écrit en 1919, il a été mis en musique par Marie-Joseph Erb (Matthis $1957: 41-42$; 199).

6 Pseudonyme de Curt Krüger, psychiatre (1897-?) umchu sin, et encore uniquement dans l'anthologie intitulée Neue Fahrt (1929: 52).

Ces anthologies montrent surtout une absence criante : ni la souffrance des soldats, ni les tourments et la vie difficile des civils, ni le patriotisme francophile tonitruant de 1919 remplissant le recueil Blöj-wiss-rot ou l'amertume d'un changement qui apporte une vie conflictuelle n'y figurent.

Serait-ce parce que les écrivains auraient été muets sur ces sujets ? Une revue de détail montre qu'ils ne sont pas nombreux à avoir évoqué, d'une manière ou d'une autre, cette période. Était-ce parce qu'il s'agissait, concernant la guerre, de blessures encore ouvertes, de l'impossibilité de mettre des mots sur l'indicible ? Pour essayer de ne rien raviver ? Pour tenter de tourner une page ? Par crainte de ne pas paraître comme un bon patriote français en ces temps d'après-guerre particulièrement complexes et conflictuels ? Pour éviter une exploitation par les acteurs des conflits politiques sévères qui allaient agiter la société alsacienne durant une bonne quinzaine d'années ? Ou étaitce tout simplement parce que la guerre ne présentait plus d'intérêt pour les auteurs (et/ou leurs lecteurs) dans un contexte nouveau?

Trois moments spécifiques auraient pu pousser à l'écriture :

- la guerre vécue de l'intérieur ou non, comme soldat ou non,

- le positionnement par rapport à la guerre en soi et le pacifisme qui en est alors fréquemment son corolaire,

- les conséquences de la guerre : le "grand tournant » de 1918 comme conséquence centrale, au moins sur le plan des appartenances nationales ou des devenirs sociaux, vu de façon positive ou négative.

\subsection{PRÉSENCE DE LA GUERRE EN TANT QUE TELLE}

\subsubsection{Nathan Katz}

C'est dans des textes publiés en 1930 que la guerre est présente en soi dans l'œuvre de Nathan Katz. En évoquant la mort d'un ami, il décrit les circonstances de cette mort :

\author{
Sterbend hingst du im Stacheldrahtzaun. \\ Die durstende Erde trank dein Blut. \\ Dein Auge brach. \\ Dein Antlitz verzerrte sich. \\ $[\ldots]$ \\ hineingestorben aus Wahnsinn und Grauen und Schützengraben \\ in den grossen Klang der Sternennächte, \\ in das verwunderte Erwachen kleiner Blüten, Freund, \\ in das Wunder sich ewig verjüngender Welt. \\ (Die Stunde des Wunders p. 60)
}

Et la même année paraît Sundgäu. Gedichter, où le cycle D'Unrüehj in de Nacht va à la fois radicaliser son propos 
autour de la guerre et clore, avec ces poèmes, presque définitivement, cette thématique dans son œuvre :

Büre, vo äiere Acker het er [dr Chrieg] ech furttribe.

Wàber, äieri Wabstiehl hait dr miesse loh steh;

D'Flinte hait dr in d' Hang gnu;

Märder sind dr worde.

's Fir hait dr in aremi Hitte $n$ ineglegt. (p. 11-12)

L'accusation terrible, sans appel, qu'il porte contre ses semblables d'être devenus des meurtriers et des incendiaires dont d'autres petites gens comme eux sont les victimes tombe comme un couperet, cette absurdité lui est insupportable, incompréhensible.

Et son poème, D'Kamerade, wu im Chrieg umchu sin, semble signer la fin d'un travail thématique littéraire, mais porte visiblement la permanence du souvenir de l'absurdité meurtrière de tous ces soldats (Frind un Find) qu'elle a emportés, pour n'en laisser que les os, jusqu'à leur disparation complète :

I sitz un dànk. - Jetz lige d' Spothärbschtnàcht

So ficht üf alle Gräber: Frind un Find,

un d' Wirem nage jetz am Totebei

vo alle, all wu umchu sin im Chrieg,

im lange, bese, färchterlige Chrieg;

sie nage jetz an allem Totebei.

Ich seh'n ech all jetz vor mr, Kamerade,

Dir sind so lang scho tot! - (p. 15)

Le pacifisme, l'entente entre les peuples, la compréhension mutuelle, la douleur humaine universelle, Katz les a thématisés très tôt, lorsqu'il était en Russie. Dans les deux petits ouvrages en allemand, d'une centaine de pages chacun, où alternent prose et poèmes, Das Galgenstüblein. Ein Kampf um die Lebensfreude et Die Stunde des Wunders, parus respectivement en 1920 et 1930, Nathan Katz partage avec le lecteur à la fois l'état d'esprit qui l'anime, mais aussi les réflexions que son état de prisonnier de guerre en Russie lui inspire. D'une part, la conviction de la nécessité de la paix comme art de vivre, la connaissance et la curiosité de l'autre ainsi que la fin des patriotismes nationalitaires semblent s'ancrer très profondément en lui. D'autre part, il affirme sa foi en l'homme, la proximité fraternelle qu'il ressent avec les paysans russes, qui ont les soucis communs à tous les paysans, l'intérêt qu'il porte à des traditions qui lui semblent proches, à la langue dont il apprend des bribes et qui lui permet de leur parler ainsi que son empathie pour tous ses camarades, prisonniers comme lui, angoissés par l'avenir qui leur est réservé, inquiets pour leur famille, leur devenir, leur vie. Il partage tout cela avec eux et tous ceux qui sont dans leur situation, quels qu'ils soient.

Dans le texte qui donne le sous-titre au recueil (Ein Kampf um die Lebensfreude), Katz s'émerveille, s'étonne et émet des souhaits :

Denke dir eine Mutter, die sich über die Wiege ihres Kindleins neigt; in ihren Augen das stolze junge Glück des Besitzens, und spreche [sic] mir sie nach die Worte: „Ono psi!“ ... „Es schläft“... Spreche [sic] es mir nach: „il dort!“... Du wirst fühlen, hier ist etwas, das ist Gesetz der Menschheit überhaupt!... Mutter!... Kind!... Hier ist eines der Bande, das ist über jedes Nationalitätsgefühl erhaben!...

Und nun: Weil du mit einer Mutter eines fremden Volkes mitempfinden konntest, weil eine Liebe zu jenem Volke in dir zu leben beginnt, wirst du deiner Heimat Volk gegenüber weniger patriotisch fühlen?!

Muss sich dir nicht die Frage aufdrängen: Verdankst du nicht diesem fremden Volke vieles, was du geworden bist?! Ja, hat nicht auch dieses fremde Volk an der Kulturentwicklung deines Volkes mitgearbeitet? (p. 47-48)

Et il conclut, en optimiste indécrottable, en plein milieu de la guerre: « Es liegen schon einzelne hohe Worte in den Munden: „Weltfrieden!“ „Völkerbund“. Der alte Fanatismus liegt zwar noch lastend auf den Massen, aber die Welt geht besserer Zeit entgegen! (p. 52) $)^{7}$ »

\subsubsection{D'autres auteurs}

Dans son recueil de poésies de 1919, D'r Lüser, qu'il divise en cinq grandes parties, Philippe Oberlé (1894-1934) intitule certes la première partie "Widder franzeesch », mais dans la section qui suit, Kriejssouvenir (p. 23-38), il montre toute l'horreur de la guerre dans Trummelfier (p. 32), même si sa langue est fortement imprégnée par la langue standard et s'il s'agit d'un texte de « circonstance » :

Wahnsinn, Wahnsinn, Menschemorde,

Menschemaßzerfleischereij.

Un in d'Nacht nin dringt so mancher

Letschter, armer Todesschreij.

Als ob töisigi Gewitter,

als ob d'Hell wär uffgemacht

un der Teifel, sini Großel,

do dezwische hoehnisch lacht.

[...]

Un es blitzt un zuckt am Himmel.

"Herr", sifftzt mancher, „geh, hilf mir,

B'schirm mich in dem Wahnsinnstobe,

b'schirm mich in dem Trummelfier". (p. 32-33)

De ce vécu de la guerre, Jean-Marie Schelcher tirera, en 1992, une sorte de "docufiction », en dialecte, ... damit Du weisst, dass ich noch lebe. Briefe eines tapferen Elsässer Soldaten aus dem 1. Weltkrieg, en s'appuyant sur l'ensemble des cartes postales qu'un de ses grands-oncles a envoyées

7 D'autres poètes plaident aussi pour une paix entre les hommes, pour la fin de la guerre ou de toutes les guerres. Dans la plupart des cas, c'est l'aspect humain, le destin familial et humain qui les amènent à ce type de demandes, qui n'est pas spécifique à l'Alsace, par ex. : Muller 1922, partie " Krieg un Friede » (p. 73-92), en particulier le poème de 1915 Das isch dr Krieg (p. 75-76) ; Voeltzel 1923 : partie " Üs m Krieg » (p. 41-50), en particulier les poèmes Im Kornfeld ; Gröisamer Kriej (p. 49-50); Zumstein Charles [s.d]) Kriegsgedichte 1914-1918, le texte Mahnruf an die Völker Europas (p. 78-79). 
à sa fiancée et sur la tradition orale qu'il a recueillie dans les deux familles. II ne s'agit pas d'un journal, mais bien de questions que quelques rares autres écrivains se sont posées sur le sens de la guerre et le rôle du soldat, " naïvement ", à hauteur d'homme : "Wià soll e Franzos oder e Anglander in mer sine Fend sàh, àr kànnt mech jo net. Dà weiss nitt vu mer. Soll ich ehm sage, ich sëig dr Bixel Thedor vun Balgäu un heig dr Uftrag vu mim Kàiser, uf ehne z'schiasse? » (p. 24) Et la guerre va transformer des hommes qui avaient été sensibles et humains :

Bi eim Dode isch mà noch betriàbt. Bim zweite nemmt 's scho ab. Bim hundertschte oder toisigschte fühlt mà ewerhäupt nitt meh... Do lüagt mà nur noch, denn mà làbt jo grad met dà Kadawer zàmme un isch so stark abghärtet, ass mà sàlbscht bim Wàltuntergang hechschtàfalls dàtt stüne. (p. 50)

\subsection{La guerre par la narration, de " seconde main »}

Ce sont plutôt dans les pièces de théâtre en dialecte que la complexité et les intrications multiples de la guerre et de ses conséquences sont évoquées. Le plus étonnant cependant, c'est qu'aucune des anthologies ne les mentionne, en particulier celle de 1933, dont l'éditeur pouvait avoir connaissance de l'ensemble des textes. II est possible qu'aux yeux des éditeurs, les critères artistique et littéraire n'aient pas été remplis. Si la pièce de Bopp et de Boesch Zwischa Fier un Liacht (Dammerung) présente de très nombreuses faiblesses (les deux auteurs ne sont pas vraiment des dramaturges) et celle de Marcel-Edmond Naegelen [1892-1978] « Vermisst » est bien trop convenue, la pièce du même Naegelen D'Ferme Iltis, publiée en 1931, est bien plus ancrée dans une sorte de plausibilité sociétale et pouvait mieux correspondre à la complexité vécue par les spectateurs. Que ce soit Naegelen qui soit l'auteur de la pièce a dû déconcerter plus d'un de ses confrères. Fils d'optant, ayant fait la guerre du côté français, membre de la SFIO, homme politique, on pouvait s'attendre à ce qu'il défende le point de vue français, d'une part, et qu'il montre peu d'empathie pour les complexités alsaciennes, comme c'est plutôt le cas dans "Vermisst", d'autre part. Les trois actes se déroulent respectivement en juillet 1914, en septembre et en novembre 1918. L'auteur pose rapidement un cadre où, en cas de guerre, il pourrait y avoir une tension potentielle entre un jeune ami de la famille, Jean, qui est Français, et la famille Iltis, qui est alsacienne, donc allemande. Le père de famille et fermier, désabusé, constate que le peuple, d'un côté comme de l'autre, est versatile et que personne ne veut la guerre, mais que tous y participeront (p. 12). Si les soldats ont des difficultés à « raconter » ce qu'ils ont vécu, la fille de la famille force la main à son frère, soldat en permission, à lui parler de sa vie de soldat. II lui raconte alors l'horreur, l'enfer, les morts (1931: 33$)$, et des soldats qui, même s'ils ne voulaient pas cette guerre, craignant pour leur propre vie, tuent sans état d'âme (1931 : 32). Aucun n'en sort indemne : " Un doch bin ich noch d'r namli ass friähjer, nur do drinne isch alles wund un weh. » (p. 34) analyse-t-il. En écho, dans le $3^{\mathrm{e}}$ acte, Jean explique aussi cette impossibilité de l'oubli :

Nie wur ich de Kriej vergesse ! Un ich will ne nit vergesse ! So lang ich läb, will ich mir vor Aue füehre, was der Kriej füer Elend angericht het; ich will dran denke, füer dass ich ne hasse kann, de Kriej, - dass ich ne wittersch pflanze kann, der [sic] Hass vum Kriej, vum rucksichtslose Massemord. [...] Anna, dü waisch nit, was so e Kriej üs eim macht... (p. 59).

\subsection{La ligne d'horizon de la paix intérieure : I'Alsace}

Si l'on repense à la centaine d'auteurs évoqués, liste dont Naegelen est absent, et le peu de cas qui semble avoir été fait de la guerre, il faut s'interroger sur les thématiques qui ont été retenues par les écrivains. Un élément central pourrait consister dans le fait que, de manière plus fréquente encore qu'avant 1914 , la question du positionnement ethno-politique et de l'appartenance nationale (France ou Allemagne) semble connaître une autre réponse : c'est l'appartenance à la « petite patrie » qui l'emporte largement, l'Alsace, célébrée sur tous les tons, qui semble être la ligne d'horizon presque exclusive de la plupart des écrivains, que ce soit dans le lyrisme ou sur scène, même si, pour certains, l'Alsace est le dernier élément auquel on peut se raccrocher lorsque le désespoir semble total :

Menschenfetzen, Drahtverhaue, Steine, Baumstümpfe fliegen durcheinander durch die Luft. [...] Die Menschheit brüllet auf im Wahnsinnsschmerz, [...] doch machtlos ist sie gegen diese Menschenhöllen, Höllenteufel.

Wahnsinn, wohin ich mich auch drehe, ringsum ein Tollhaus, rundum Hölle. [...]

Ich möchte schreien, doch die Stimm' erstickt.

Ich möchte fliehen, doch die Beine zittern. [...]

Doch aus dem Dunkel der Umnachtung und des Wahnsinns steigt ein Bild in lichtem Glanz von weit, aus einer andern Welt zu mir hernieder:

Heimat ... Elsaß!

Mutterantlitz ... Vaterhaus!

(Oberle, [1928] : 53-54, poème Aus Tod und Not

[Verdun, 1916])

D'une manière ou d'une autre, ils ont presque tous évoqué l'Alsace, par le biais de la nature et les paysages, par les villes et les villages, par le sentiment amoureux, par les us et coutumes, par les activités des hommes, mais aussi par la langue et par l'identité qu'elle confère à ses habitants. Par ailleurs, il est frappant de voir que les écrivains prolixes et populaires comme Ferdinand Bastian (1868-1944), certes auteur de comédies, mais aussi de Volksstücke (en dialecte) d'une certaine tenue avant 1914, ne publie plus que des textes destinés à faire sourire ou rire les lecteurs ou spectateurs après la guerre. Son cadet Fritz Stephan (1884-1961), surnommé 
" de Schnirichle Fritz », narrateur d'histoires drôles, homme de théâtre et de radio, portant un regard amusé sur la société et ses travers au quotidien, suit le même chemin ${ }^{8}$ L'humour non politique serait-il un antidote à la gravité de l'air du temps ? Un moyen de s'en échapper ou de le rendre plus supportable ${ }^{9}$ ?

Cette littérature ne garde que peu de traces de la guerre et de ses conséquences (ou alors essentiellement en creux) sauf si l'on considère que le centrage sur l'Alsace, comme valeur-refuge, comme espace matriciel construit dans les discours depuis la fin du $\mathrm{XIX}^{\mathrm{e}}$ siècle et alimenté depuis lors par une recherche patrimoniale justifiant une sorte d'Alsace éternelle est précisément la conséquence majeure à la fois

8 Les quatre anthologies ne mentionnent ni l'un ni l'autre, excepté celle de Schaeffer qui évoque un "Prolog » écrit par Bastian pour la réouverture du Théâtre Alsacien de Strasbourg. II précise, à propos de Bastian : « Es betritt nicht gern jeder Dichter den politischen Boden. " (Schaeffer $1919: 2$ )

9 C'est ce qu'il semble penser dans la plaquette qu'il publie en 1918: « Mini Absicht isch, Euch e bissel Heimetluft ze schicke, die Euch e Stüendel Gemüetsrüehj verschaffe soll. (Stephan 1918:1) de la guerre et de ses suites immanentes. Ce centrage sur l'Alsace permet de contourner la guerre, mais se trouve aussi totalement en phase avec le discours sociopolitique circulant dans la société durant l'entre-deux-guerres. II s'agit d'une construction représentationnelle qui confère une singularité par rapport aux États, à la fois atemporelle et inscrite dans des signes tangibles non littéraires (culturels, religieux, linguistiques, ...).

Un travail plus approfondi de l'ensemble des publications des auteurs cités, mais aussi et surtout un examen précis des comptes rendus dans la vaste palette de la presse entre 1918 et 1939 , y compris la presse satirique, permettraient de mieux comprendre la rareté des traces qu'aura laissé la Grande Guerre en soi dans la littérature en Alsace. II n'est pas impossible que le Second conflit mondial et l'annexion de fait de l'Alsace à l'Allemagne nazie (1940-1945), plus traumatiques encore, aient contribué à jeter, par la suite, les traces de la Grande Guerre dans les oubliettes de la mémoire.

\section{Bibliographie}

\section{Anthologies}

Buchert, Raymond (Hg.) (1929), Neue Fahrt. Elsässische und lothringische Lyrik der Nachkriegszeit, Gebweiler, Alsatia-Verlag (Elsaßland-Bücherei - Band 7).

Reinacher, Eduard (Hg.) (1925), Das Elsässer Schiff mit Fracht von Bildern und Liedern, Stuttgart, W. Hädecke.

Schaeffer, Louis Edouard (Hg.) (1919), Blöuj-Wiss-Rot. Eine Sammlung neuer elsässischer Dialektgedichte aus den Tagen der Befreiung als Ergänzung zum Neuen Elsässer Schatzkästel, Strasbourg, Imprimerie Strasbourgeoise.

Solveen, Henri (éd.) (1924), L'Arc. Anthologie publiée par l'Arc, Strasbourg, Les Éditions de l'Arc.

Wackenheim, Auguste, La littérature dialectale alsacienne.

- Tome 3 (1997), La période allemande, 1870-1918, Paris, Prat-Éditions.

- Tome 4 (1999), D'une guerre mondiale à l'autre, 1918-1945, Paris, Prat-Éditions.

Walter, Karl (Hg.) (1933), Zwischen Rhein und Mosel. Elsässische Dichtung der Gegenwart, Straßburg, Heitz Verlag.

\section{Cuvres citées}

Bopp, Marie-Joseph \& Boesch, Georges (1920?), Zwischa Fier un Liacht (Dammerung). Elsassisch Schoispeel en vier Akte üss der Zit noch dr Armistice, Colmar, Imprimerie Strasbourgeoise.

Katz, Nathan (1920), Das Galgenstüblein. Ein Kampf um die Lebensfreude, Strasbourg/Mulhouse, Édition de "La littérature populaire ".

Katz, Nathan (1930), Die Stunde des Wunders, Gebweiler, Alsatia-Verlag (Elsass-Bücherei - Band 10).

Katz, Nathan (1930), Sundgäu, Colmar, Alsatia.

Matthis, Albert \& Adolphe (1931), Aephai, Stroosburri, Imprimerie des Dernières Nouvelles de Strasbourg.
Matthis, Albert \& Adolphe (1957), Bissali. Augmenté d'un choix d'Aephai, de Fülefüte et d'inédits pour le $35^{\circ}$ anniversaire de la première édition, avec une introduction et des notes par Alfred Schlagdenhauffen, Paris, Les Belles Lettres.

Müntzer, Désiré ([1913]) Neues Elsässer Schatzkästlein. Eine Sammlung elsässischer Dialektgedichte aus Vergangenheit und Gegenwart, Straßburger Druckerei und Verlagsanstalt, Straßburg i. E.

Muller, Henri (1922), Zittelposcht. Gedichtle in dr Heimetsproch, Mulhouse, Librairie centrale.

Naegelen, Marcel-Edmond (1930), "Vermisst ». Dramatischi Skizz in 1 Akt, Strasbourg, Édition des Dernières Nouvelles de Strasbourg.

Naegelen, Marcel-Edmond (éd. 1949 [1931]), D'Ferme Iltis. Drama in 3 Akt, Gundershoffen, Jaggi-Reiss.

Oberlé, Philippe (1919), D'r Lüser. Elsässischi Gedichte üs guete un bese Stunde, Straßburg, Selbschtverlag des Verfassers.

Oberlé, Philippe (1928), Elsässische Heimat, Strassburg-Neudorf, Erwinia.

Schelcher, Jean-Marie (1992), ... damit Du weisst, dass ich noch lebe. Briefe eines tapferen Elsässer Soldaten aus dem 1. Weltkrieg, Basel, Janus Verlag.

Stephan, Fritz (1918), Geschichtle üs d'r Heimet for mini Landslitt im Kriej, Strassburg, Vomhoff.

Voeltzel, L. W. (1923), Heimgedenke. Gedichte in Elsässer Mundart, Strassburg, Buchhandlung der Evangelischen Gesellschaft.

Zumstein, Charles (s.d.), Kriegsgedichte 1914-1918, Mulhouse, Librairie centrale.

\section{Littérature secondaire citée}

Baas, Geneviève (1972), Le malaise alsacien 1919-1924, Strasbourg, Développement et communauté. 
Baechler, Christian (2008), Les Alsaciens et le Grand Tournant de 1918, Strasbourg, L'Ami Hebdo/M.E.D.I.A.

Fichter, Charles (2010), Pour une autre histoire de la littérature alsacienne au début du XX $X^{e}$ siècle. Loin de la nostalgie et du ressentiment, Strasbourg, bf.

Götze, Alfred (1917), Das Elsaß und die poetische Literatur des Weltkriegs. Vortrag gehalten in der Hauptversammlung am 8. März 1917, Straßburg, Karl J. Trübner (Einzelschriften zur Elsässischen Geistes- und Kulturgeschichte, 3).
Grandhomme, Jean-Noël et Francis (2013), Les Alsaciens-Lorrains dans la Grande Guerre, Strasbourg, La Nuée Bleue.

Stoskopf, Gustave (2019), Un étudiant alsacien à Paris, 1887-1894. Correspondance familiale et récit autobiographique sur la vie d'artiste, Eckbolsheim, Éd. du Signe.

Stoskopf, Nicolas (2019), « Gustave Stoskopf et l'identité alsacienne », Land un Sproch. Les cahiers du bilinguisme 211, septembre 2019, 8-9.

Wahl, Alfred (2019), Les autonomistes en Alsace, 1871-1939, Orbe, Éd. du Château. 